Revista Tecné, Episteme y Didaxis: TED. Año 2014, Número

Extraordinario. ISSN Impreso: 0121-3814, ISSN web: 2323-0126

Memorias, Sexto Congreso Internacional sobre Formación de

Profesores de Ciencias. 08 al 10 de octubre de 2014, Bogotá

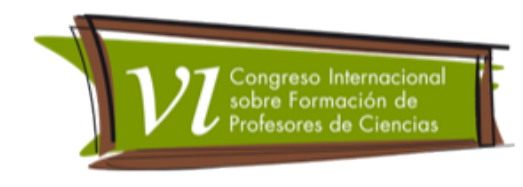

\title{
Construcción de los conocimientos de los futuros profesores de ciencias en un espacio de formación alternativo
}

Rengifo Gallego, Luz Adriana'

Categoría 2: Trabajo de investigación en proceso

\section{Resumen}

Este documento presenta un proyecto de tesis doctoral que pretende, a través de un espacio de formación alternativo, contribuir a que los profesores de ciencias naturales en formación inicial de un programa curricular, identifiquen y desarrollen sus conocimientos profesionales integrados, de manera que les permita el abordaje de problemas complejos en la educación básica.

\section{Palabras clave}

Formación inicial de profesores, conocimiento profesional del profesor, problemas complejos.

\section{Introducción}

Esta investigación se desarrolla en el contexto del programa curricular Licenciatura en Educación Básica énfasis en Ciencias Naturales y Educación Ambiental de la Universidad del Valle (Cali-Colombia). Dicho programa manifiesta, por una parte buscar la articulación entre las Ciencias (disciplinas) y la Pedagogía (enseñanza, aprendizaje y evaluación) a través de los diferentes cursos repartidos en cuatro componentes: científico, pedagógico, didáctico y socio-ambiental; pero por otro lado los cursos de estos componentes se encuentran separados, ofertados en facultades diferentes, sin mayor interconexión en sus objetivos y sus contenidos.

Así, se aborda el problema de la fragmentación de conocimientos por parte de los futuros profesores, que se produce en modelos de formación tecnológicos (Porlán y Rivero, 1998; Feiman-Nemser, 1990) donde, como es este caso, se yuxtaponen los conocimientos científicos (biología, química, física...) y conocimientos para la enseñanza. Estos conocimientos fragmentados dificultan

I Universidad del Valle. luzareng@hotmail.com 
Revista Tecné, Episteme y Didaxis: TED. Año 2014, Número Extraordinario. ISSN Impreso: 0121-3814, ISSN web: 2323-0126

Memorias, Sexto Congreso Internacional sobre Formación de Profesores de Ciencias. 08 al 10 de octubre de 2014, Bogotá

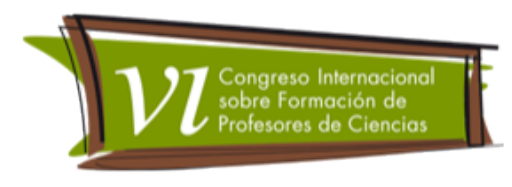

abordar en la educación básica escolar problemas complejos, como los problemas actuales que involucran situaciones sociales, culturales y políticas, manifiestas en diversos planos como por ejemplo la salud, planificación del territorio, marginación de las minorías, mejoras de la calidad de vida, control demográfico, que requieren conocimientos de diferentes disciplinas para su abordaje y solución (García, 1998; y Morin, 2002; Sadler, 2004; Levinson, 2008).

Shön (1987) menciona que desde esta perspectiva de formación se defiende la idea de que los profesionales de la práctica, en este caso los profesores, solucionan problemas instrumentales mediante la selección de los medios técnicos más idóneos para determinados propósitos. Este modelo se sustenta en la división clásica entre teoría y práctica, donde dicha práctica se concibe como la mera aplicación de las teorías sobre la enseñanza y el aprendizaje.

Martín del Pozo, et al (2013) plantean que desde este modelo lo primero es dar a los profesores en formación una perspectiva académica de los contenidos de la materia a enseñar, posteriormente una formación profesional sobre los conocimientos pedagógicos y las destrezas sociales y comunicativas que se necesitan para enseñar los contenidos en el contexto escolar.

Este tipo de modelo constituyó según Shön (1987) los currículos normativos de las primeras décadas del siglo XX hasta su época. Además puede considerarse un modelo de formación vigente actualmente en Colombia, ya que si bien dentro de los programas universitarios se ha intentado combinar en un mismo semestre cursos disciplinares referidos a la materia a enseñar y cursos referidos a cómo enseñar, en algunos casos los cursos de un programa son ofrecidos por dos facultades distintas: una de Ciencias donde se enseñan los conocimientos disciplinares de la materia y otra de Ciencias de la Educación o de Educación y Pedagogía ${ }^{2}$, en ocasiones sin la vinculación curricular necesaria entre ellas que promueva una sincronización entre los propósitos, contenidos y metodologías de trabajo entre los cursos.

En este sentido, frente al problema de la fragmentación del conocimiento en el que se forman los futuros profesores en el programa curricular en mención, surge entonces la necesidad de dar respuesta al siguiente interrogante:

\footnotetext{
${ }^{2}$ Puede presentar diferentes denominaciones según la Universidad
} 
Revista Tecné, Episteme y Didaxis: TED. Año 2014, Número Extraordinario. ISSN Impreso: 0121-3814, ISSN web: 2323-0126

Memorias, Sexto Congreso Internacional sobre Formación de Profesores de Ciencias. 08 al 10 de octubre de 2014, Bogotá

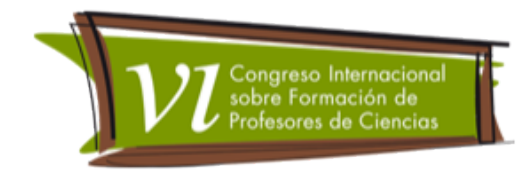

¿Qué conocimiento profesional construyen los futuros profesores de ciencias naturales cuando participan en un espacio de formación inicial alternativo que presenta el conocimiento profesional integrado?

\section{Objetivos}

El objetivo general de esta investigación es contribuir a que los profesores en formación inicial identifiquen y desarrollen sus conocimientos profesionales de manera que les permita el abordaje de problemas complejos en la educación básica.

Los objetivos específicos son:

- Diseñar, desarrollar y evaluar un espacio de formación inicial alternativo que integre los conocimientos del profesor necesarios para enseñar ciencias mediante la investigación escolar de problemas complejos.

- Describir y analizar los conocimientos profesionales iniciales de los profesores participantes en un espacio de formación inicial alternativo de epistemología integradora.

- Describir y analizar el desarrollo de los conocimientos profesionales de los profesores participantes en un espacio de formación inicial alternativo que integra el conocimiento profesional del profesor.

\section{Marco teórico}

El marco teórico de esta investigación, supone referirse al conocimiento del profesor. Dentro de las conceptualizaciones del conocimiento del profesor para la enseñanza se pueden identificar básicamente tres tendencias: la primera de ellas reconoce el Conocimiento Pedagógico del Contenido como núcleo fundamental del conocimiento del profesor, la segunda centra el conocimiento profesional del profesor en su Conocimiento Práctico Personal, y la tercera concibe el conocimiento del profesor como una Integración de Conocimientos de naturalezas diferentes.

En la primera de dichas tendencia se encuentra el modelo de Shulman (1987), continuado y modificado posteriormente por otros autores como Grossman (1990) y Magnusson, Krajcik, y Borko (1999). Los primeros trabajos al respecto fueron publicadas como: "Those Who Understand: Knowledge Growth in Teaching" (1986) y "Conocimiento y Enseñanza" (1987) donde Shulman identifica la 
Revista Tecné, Episteme y Didaxis: TED. Año 2014, Número Extraordinario. ISSN Impreso: 0121-3814, ISSN web: 2323-0126

Memorias, Sexto Congreso Internacional sobre Formación de Profesores de Ciencias. 08 al 10 de octubre de 2014, Bogotá

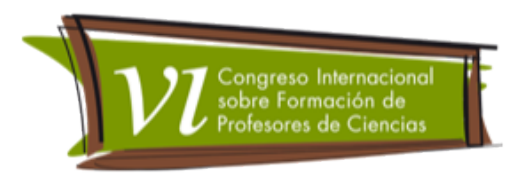

necesidad de reconocer las características del conocimiento del profesor y fundamentar la necesidad de reorientar el concepto de la enseñanza, al igual que la formación y evaluación de los profesores, en función de lo que debería contemplar la "base de conocimientos para la enseñanza".

Desde ahí se determina el conocimiento Pedagógico del Contenido (PCK por sus siglas en ingles Pedagogical Content Knowledge) como el conocimiento fundamental del profesor. Éste se define como la mezcla entre materia y pedagogía; por esta mezcla se llega a una comprensión de cómo determinados temas y problemas se organizan, se representan y se adaptan a los diversos intereses y capacidades de los alumnos, y se exponen para su enseñanza (Shulman, 1987). Así, "el conocimiento pedagógico de la materia es la categoría que con mayor probabilidad permite distinguir entre la comprensión del especialista en un área del saber y la comprensión del pedagogo" (Shulman, 1987, pág.13).

Desde esta tradición, el conocimiento que produce el profesor es un conocimiento "profesional" referido a cómo enseñar el contenido disciplinar producido por comunidades académicas (comunidades de científicos) externas al aula. El qué enseñar del profesor, es el conocimiento científico disciplinar, el cual es de naturaleza externa al aula. El conocimiento que produce el profesor es la organización y representación de ese conocimiento científico disciplinar, de ahí que el interés se centre en recoger las analogías, metáforas, ejemplos, demostraciones y explicaciones que el profesor produce sobre ese conocimiento a enseñar. Sin embargo desde los planteamientos de autores como Rodrigo (1997), Zambrano (1998), García (1998), Perafán (2012) el conocimiento que enseña el profesor en el aula es un conocimiento escolar, caracterizado por poseer una naturaleza diferente al conocimiento científico, que de acuerdo con Chevallard (1991) tiene su objeto no en la disciplina (por ejemplo matemática), sino en las prácticas sociales de ella, donde "se afirma una intención de aprender o enseñar un objeto de saber..." (Chevallard, 1991, pág. 174).

La segunda de las tendencias del conocimiento del profesor, aquella que centra el conocimiento profesional del profesor en su Conocimiento Práctico Personal, conceptualiza el conocimiento del profesor como un "conocimiento local" generado por los propios profesores en sus contextos locales de trabajo. Autores como Connelly, Clandinin y He (1997) entienden el conocimiento de los maestros como derivado de la experiencia personal, de modo que no es algo objetivo e 
Revista Tecné, Episteme y Didaxis: TED. Año 2014, Número Extraordinario. ISSN Impreso: 0121-3814, ISSN web: 2323-0126

Memorias, Sexto Congreso Internacional sobre Formación de Profesores de Ciencias. 08 al 10 de octubre de 2014, Bogotá

independiente del profesor para ser aprendido y transmitido, sino con un enfoque más cualitativo y etnográfico, corresponde a la suma total de las experiencias pasadas y las intenciones futuras del profesor que le permiten hacer frente a las exigencias de la situación actual. En términos de Carter (1990) el conocimiento práctico es aquél que "los docentes tienen de las situaciones de clase y de los dilemas con los que se enfrentan actuando propositivamente en dichos ámbitos" (Carter, 1990. pág. 299).

Pero pensar el conocimiento del profesor desde el conocimiento práctico personal implicaría una primacía del saber basado en la experiencia no solo profesional sino también personal, frente al saber académico y tecnológico (la dimensión práctica de la actividad docente es el resultado técnico de una aplicación de la teoría), superponiendo la acción sobre la reflexión.

Reconociendo la importancia tanto del referente del conocimiento de la disciplina a enseñar y el conocimiento que permite la enseñanza, entre ellos el conocimiento práctico personal, pero identificando además las características propias del conocimiento del profesor que resulta de la integración de estos conocimientos de naturalezas diferentes, se encuentra una tercera tradición.

La tercera de las tendencias que concibe el conocimiento del profesor como una Integración de Conocimientos de naturalezas diferentes, recoge otra conceptualización y otro marco teórico del conocimiento del profesor diferente al de Shulman (1987), donde el conocimiento del profesor no solo incluye como enseñar el conocimiento producido por comunidades académicas científicas, sino que contiene lo referente a qué se enseña. Desde esta tendencia se han presentado investigaciones interesadas en la identificación del conocimiento profesional deseable (Porlan y Rivero, 1998) y el conocimiento profesional docente específico de los profesores (Perafan, 2004), dándole un papel importante dentro de este a lo que algunos autores (Pajares, 1992; Connelly et al. 1997; Jones y Carter, 2007) han denominado creencias del profesor, las cuales se refieren a opiniones, valores, actitudes que corresponden a un conocimiento implícito.

Este conocimiento profesional del profesor se caracteriza por la propiedad de emergencia, es decir que es poseedor de unas características diferente a los conocimientos que lo conforman y responde a problemáticas diferentes a las que responde cada uno de los conocimientos que lo componen. 
Revista Tecné, Episteme y Didaxis: TED. Año 2014, Número Extraordinario. ISSN Impreso: 0121-3814, ISSN web: 2323-0126

Memorias, Sexto Congreso Internacional sobre Formación de Profesores de Ciencias. 08 al 10 de octubre de 2014, Bogotá

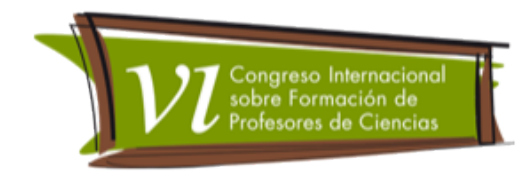

\section{Metodología}

Para el problema de investigación señalado, se lleva a cabo un estudio de casos desde un enfoque interpretativo, donde se reconocen (de acuerdo con Carr y Kemmis, 1988) los participantes de la investigación como sujetos con estructuras intrínsecamente significativas, constituida y sostenida por sus actividades interpretativas diarias, que constituyen la realidad. En coherencia con ello será el trato que se le dará a la recolección, análisis e interpretación de la información.

El contexto para esta investigación es un espacio de formación alternativo en el programa curricular Licenciatura en Educación Básica Énfasis en Ciencias Naturales y Educación Ambiental de la Universidad del Valle (Cali - Colombia), la cual se escoge por ser una de las principales universidades acreditadas del Suroccidente Colombiano para ofrecer este programa en el país, porque su programa manifiesta en su concepción del conocimiento algunas características del modelo de formación tecnológico, por la necesidad manifiesta desde las directivas del programa de restructuración de éste, así como por el acceso a él.

La propuesta de formación inicial alternativa se caracteriza por tener como núcleo central la actividad práctica de enseñar. Alrededor de ella se organizan tres contextos de reflexión y desarrollo: 1) la enseñanza de profesores en ejercicio, 2) los aportes de los expertos del campo de la Educación en Ciencia, 3) la propia enseñanza.

En cada uno de ellos se abordan integradamente los conocimientos profesionales del profesor de ciencias, referidos a cada uno de sus componentes: conocimiento del contenido, conocimientos didácticos, prácticos personales y contextuales. El punto de partida es el planteamiento de la propuesta de enseñanza de los futuros profesores, la cual se modificará a lo largo del curso, según los aportes brindados.

La muestra de esta investigación estará formada por los participantes (estará sujeto al número de matriculados, entre 8 y 25 personas) en el espacio de formación inicial alternativo en el marco del programa curricular en mención. Estos profesores son un caso $\theta$ que constituyen un objetivo particular como sistema acotado (Stake, 1999) para permitir su estudio dentro de las posibilidades de espacio y tiempo. 
Revista Tecné, Episteme y Didaxis: TED. Año 2014, Número Extraordinario. ISSN Impreso: 0121-3814, ISSN web: 2323-0126

Memorias, Sexto Congreso Internacional sobre Formación de Profesores de Ciencias. 08 al 10 de octubre de 2014, Bogotá

La investigación se llevará a cabo en un proceso de cinco fases:

1. Diseño del espacio de formación inicial alternativo.

2. Diseño y validación de los instrumentos para la obtención de los datos (cuestionarios, plantillas de análisis de los diarios de campo y las observaciones).

3. Obtención de los datos para la identificación de los conocimientos profesionales iniciales $\left(X_{0}\right)$, intermedios $\left(X_{1}\right)$ y finales $\left(X_{2}\right)$ de los futuros profesores, en el espacio de formación.

4. Sistematización y análisis de la información

5. Elaboración de conclusiones e implicaciones en la formación inicial de profesores de ciencias.

\section{Conclusiones}

Una investigación de estas características es de gran importancia para la formación inicial de profesores en nuestro país y para el campo de la Educación en Ciencias. Con esta se espera aportar al programa de formación inicial de profesores en ciencias naturales de interés (el de la Universidad del Valle) y en general a los programas de formación inicial de profesores en ciencias naturales. Se trata de aportar al desarrollo de los conocimientos profesionales de los futuros profesores de ciencias participantes en el espacio de formación y al de profesores que participen posteriormente en otros espacios de formación que tomen como modelo los principios teóricos y metodológicos de éste. Y finalmente se espera aportar a la teoría de la formación inicial de profesores de ciencias naturales.

\section{Referencias bibliográficas}

Carr, W. y Kemmis, S. (1988) Teoría crítica de la enseñanza. La investigación acción en la formación del profesorado. Barcelona: Martínez Roca.

Carter, K. (1990) Teachers' knowledge and learning to teach. En W.R. Houston (Ed.). Handbook of research on teacher education. New York: MacMillan.

Chevallard, Y. (1991) La transposición didáctica. Del saber sabio al saber enseñado. Madrid: Editorial AIQUE. 
Revista Tecné, Episteme y Didaxis: TED. Año 2014, Número Extraordinario. ISSN Impreso: 0121-3814, ISSN web: 2323-0126

Memorias, Sexto Congreso Internacional sobre Formación de Profesores de Ciencias. 08 al 10 de octubre de 2014, Bogotá

Connelly M. F., Clandinin J. D., y He, M. F. (1997) Teachers Personal Practical Knowledge on the Professional Knowledge Landscape. Teaching and Teacher Education, Vol. 13, Issue 7, pág.

Feiman-Nemser, S. (1990) Teacher preparation: structural and conceptual alternatives. En W. R. Houston (Ed.), Handbook for Research on Teacher Education. New York: Macmillan.

García, E. (1998) Hacia una teoría alternativa sobre los contenidos escolares. Serie Fundamentos N8 Colección Investigación y Enseñanza. Sevilla: Díada

Grossman, P. L. (1990). The making of a teacher: teacher knowledge and teacher education. Nueva York: Teachers College Press.

Jones, M. G. y Carter, G. (2007). Science teacher attitudes and beliefs. En S. K. Abell y N. G. Lederman (Eds.), Handbook of research on science education (págs. 1067- 1104). Mahwah, New Jersey: Lawrence Erlbaum Associates.

Levinson, R. (2008) A Theory of Curricular Approaches to the Teaching of SocioScientific Issues. ALEXANDRIA Revista de Educação em Ciência e Tecnologia, v.1, n.1, págs. 133-151

Magnusson, S., Krajcik, J., y Borko, H. (1999). Nature, sources and development of pedagogical content knowledge for science teaching. En J. GessNewsome \& N. G. Lederman (Eds.), Examining pedagogical content knowledge: The construct and its implications for science education (págs. 95-132).

Martín del Pozo, R. Fernandez, P.; Gonzalez, M.; De Juanas, A. (2013) El dominio de los contenidos escolares: competencia profesional y formación inicial de maestros. Revista de Educación, 360. Enero-abril

Morin, E. (2002) La cabeza bien puesta. Repensar la reforma. Reformar el pensamiento. Buenos Aires: Edición Nueva Visión.

Pajares, M. F. (1992) Teachers' beliefs and educational research: cleaning up a messy construct. Review of Educational Research, 62, 307-332. 
Revista Tecné, Episteme y Didaxis: TED. Año 2014, Número Extraordinario. ISSN Impreso: 0121-3814, ISSN web: 2323-0126

Memorias, Sexto Congreso Internacional sobre Formación de Profesores de Ciencias. 08 al 10 de octubre de 2014, Bogotá

Perafán, G. (2004) La epistemología del profesor sobre su propio conocimiento profesional. Bogotá: Universidad Pedagógica Nacional

Perafán, G. (2012) La transposición didáctica como estatuto epistemológico fundante de los saberes académicos del profesor de ciencias. III Congreso Internacional y VIII Nacional de Investigación en Educación, Pedagogía y Formación Docente

Porlán, R. y Rivero, A. (1998). El conocimiento de los profesores. Sevilla: Díada Editora

Rodrigo, M. (1997) El hombre de la calle, el científico y el alumno: ¿̇un solo constructivismo o tres? Novedades Educativas No. 76, págs. 59-61

Sadler, T. (2004) Informal Reasoning Regarding Socioscientific Issues: A Critical Review of Research. Journal of Research in Science Teaching. Vol. 41, No. 5, págs. 513-536.

Shön, D. (1987) La formación de profesionales reflexivos. Hacia un nuevo diseño de la enseñanza y el aprendizaje en las profesiones. Temas de Educación Madrid: Paidos

Shulman, L. (1987) Knowledge and Teaching. Foundations of the New Reform. Harvard Educational Review, Vol. 57, N 1

Stake, R. (1999) Investigación con estudio de casos. Madrid: Morata

Zambrano, A. (1998) Línea de investigación: La relación entre el conocimiento común y el conocimiento científico en el contexto de la enseñanza, aprendizaje y cambio conceptual de las ciencias. Revista Tecne, Episteme y Didaxis No. 3. Universidad Pedagógica Nacional 